\title{
An Analysis of Behaviour Change Techniques Used in a Sample of Gestational Weight Management Trials
}

\author{
H. Soltani, ${ }^{1}$ M. A. Arden, ${ }^{2}$ A. M. S. Duxbury, ${ }^{1}$ and F. J. Fair ${ }^{1}$ \\ ${ }^{1}$ Centre for Health and Social Care Research, Sheffield Hallam University, Montgomery House, 32 Collegiate Crescent, \\ Sheffield S10 2BP, UK \\ ${ }^{2}$ Department of Psychology, Sociology \& Politics, Sheffield Hallam University, Heart of the Campus, Collegiate Crescent, \\ Sheffield S10 2BQ, UK
}

Correspondence should be addressed to H. Soltani; h.soltani@shu.ac.uk

Received 4 November 2015; Accepted 3 February 2016

Academic Editor: Rosa Corcoy

Copyright (C) 2016 H. Soltani et al. This is an open access article distributed under the Creative Commons Attribution License, which permits unrestricted use, distribution, and reproduction in any medium, provided the original work is properly cited.

Introduction. Maternal obesity and excessive gestational weight gain are associated with multiple adverse outcomes. There is a lack of clarity on the specific components of effective interventions to support pregnant women with gestational weight management. Method. All 44 studies within a preexisting review of lifestyle interventions, with a potential to impact on maternal weight outcomes, were considered for content analysis. Interventions were classified using Behaviour Change Technique (BCT) taxonomy clusters to explore which categories of BCT were used in interventions and their effectiveness in managing gestational weight gain. Results. The most commonly used BCTs were within the categories of "feedback and monitoring," "shaping knowledge," "goals and planning," "repetition and substitution," "antecedents," and "comparison of behaviours." For diet and mixed interventions "feedback and monitoring," "shaping knowledge," and "goals and planning" appeared the most successful BCT categories. Conclusions. Poor reporting within studies in defining the BCTs used, in clarifying the differences in processes between intervention and control groups, and in differentiating between the intervention and research processes made BCT classification difficult. Future studies should elaborate more clearly on the behaviour change techniques used and report them accurately to allow a better understanding of the effective ingredients for lifestyle interventions during pregnancy.

\section{Introduction}

Maternal obesity and excessive gestational weight gain are associated with adverse outcomes (such as macrosomia, shoulder dystocia, and gestational diabetes $[1,2])$ and are on the rise. Despite an urgent need for evidence based guidance to support pregnant women on gestational weight management, there is a lack of clarity about effective interventions and their specific components. Interventions developed to reduce excessive gestational weight gain and its associated outcomes generally fit into the broad categories of dietary only, physical activity only, and mixed approaches utilising both diet and physical activity components [3]. It is important to identify which components and specific behaviour change techniques within these complex interventions are most effective, since this is needed to inform the development of future interventions and guidance.
Michie et al. have reported a consensually agreed structured taxonomy of behaviour change techniques which provides a framework for a more precise reporting of complex interventions [4]. The Behaviour Change Technique (BCT) taxonomy [4] is a useful tool to extract the active components of interventions, allowing comparisons between the component parts of successful and unsuccessful behaviour change interventions. Several studies [5-7] have used the behaviour change technique taxonomy described by Michie et al. [8] to define gestational weight gain management interventions. However only Currie et al. [9] have used the most up-to-date clustered BCT taxonomy [4] to code lifestyle interventions during pregnancy or the postnatal period, in their systematic review of 14 studies aimed at reducing the decline in physical activity during pregnancy.

Gestational weight management strategies often rely on complex interventions involving various interacting 
components. Identification of active components of these interventions would help in better understanding and interpreting the results of the existing systematic reviews. It would also be helpful to inform the design of new interventions and their evaluations.

Numerous systematic reviews have evaluated the efficacy of interventions designed to improve weight outcomes for mothers $[3,5,6,10-14]$. Of these most included 9 to 11 interventional studies $[5,10,11,13]$, with one review [14] only including 5 studies, two reviews including 19 studies $[6,12]$, and the final review by Thangaratinam et al. [3] of 44 studies. The reviews by Brown et al. [14], Thangaratinam et al. [3], and Choi et al. [13] focused exclusively on randomised controlled trials. Results across the reviews have varied. Streuling et al. [10] found that physical activity or diet alone interventions were not effective at reducing gestational weight gain but interventions based on physical activity and dietary counselling combined with weight monitoring appeared to be successful. In comparison Choi et al. [13] found that obese and overweight women allocated to physical activity or physical activity plus diet interventions in pregnancy had lower gestational weight gains, with supervised physical activity being especially effective. Thangaratinam et al. [3] showed some evidence of effectiveness across all interventions in reducing gestational weight gain (mean difference (MD) $-1.42,95 \%$ confidence interval (CI) -1.89 to -0.95$)$. They also reported significant reductions in weight gain in pregnancy in subgroup analysis for dietary interventions (MD -3.84, 95\% CI -5.22 to -2.45 ), physical activity interventions (MD $-0.72,95 \% \mathrm{CI}-1.20$ to -0.25$)$, and interventions with a mixed approach (MD $-1.06,95 \% \mathrm{CI}-1.67$ to -0.46 ).

Due to the comprehensive approach in inclusivity and rigour in Thangaratinam et al.s [3] review and due to it being the most highly accessed and cited article within the field of research of gestational weight management, this was selected as the source of literature for content analysis in our review. The aim of this study was therefore to evaluate the behaviour change techniques included in diet, physical activity, or mixed interventions with a potential to impact on maternal or fetal outcomes related to weight and to identify the categories of behaviour change technique of those interventions which were effective. To our knowledge, this is the first study to use the BCT taxonomy to identify techniques used in a wide range of gestational weight management lifestyle interventions.

1.1. Objectives. To explore the patterns of behaviour change techniques used in interventions with a potential to impact maternal and fetal outcomes related to gestational weight gain.

\section{Methods}

2.1. Data Selection. This study was based on the 44 randomised controlled trials of interventions with a potential to impact maternal or fetal outcomes related to weight which were included in the HTA commissioned systematic review [3]. The studies included in the review were focused on diet only ( $n=13)$, physical activity only $(n=18)$, or mixed $(n=13)$ diet and physical activity interventions for a range of pregnant women, focussing specifically on overweight and or obese women in 11 studies. The study selection criteria and assessments of quality and bias have all been reported by Thangaratinam et al. [3]. They found that the quality of studies included in the analysis for gestational weight gain was moderate, but quality for other outcomes such as preterm delivery and hypertension was low, where there may have been a risk of publication bias.

2.2. Data Extraction and Synthesis. Michie et al.s [4] health behaviour change technique taxonomy was used to identify the behavioural components of the intervention within each included study. This taxonomy contains 93 itemised health behaviour change techniques which are clustered into 16 groupings (see the following list), with each group containing between 3 and 11 clustered behaviour change techniques. For practicality of reporting the category groupings were used for the purpose of this review.

Groupings within Michie et al.s [4] Hierarchically Clustered Behaviour Change Technique Taxonomy. Consider the following:

(1) Goals and planning.

(2) Feedback and monitoring.

(3) Social support.

(4) Shaping knowledge.

(5) Natural consequences.

(6) Comparison of behaviour.

(7) Associations.

(8) Repetition and substitution.

(9) Comparison of outcomes.

(10) Reward and threat.

(11) Regulation.

(12) Antecedents.

(13) Identity.

(14) Scheduled consequences.

(15) Self-belief.

(16) Covert learning.

Three researchers (H. Soltani, M. A. Arden, and A. M. S. Duxbury) independently extracted and coded the data, to improve reliability of the data categorisation. Where there were differences in coding, the research team had a discussion to reach consensus regarding the codes and categories.

Behaviour change technique categories were classified as successful or unsuccessful within each study dependent upon whether a significant difference was found between the intervention and control group on gestational weight gain. Due to the heterogeneity of the included studies data was synthesised narratively and presented in tables and graphs as statistical synthesis was not possible. 




FIGURE 1: Behaviour change technique taxonomy categories of the interventions in included studies ( $n=44$ studies).

\section{Results}

Of the original 44 papers included within the Thangaratinam et al. review [3], one study only consisted of a conference abstract [26]. Full-text versions of all of the other articles were obtained. The 44 trials included 7627 women who had been randomised. Healthcare professionals delivering the interventions varied across the studies and included dieticians, nutritionists, clinical psychologists, doctor, nurses, and midwives.

Table 1 contains study characteristics and the behaviour change technique categories agreed by the researchers for each of the included studies [15-59]. It was not possible to apply any behaviour change taxonomy code to 10 of the studies. Figure 1 shows the distribution of BCT categories within the studies. The most commonly used behaviour change technique clusters were "feedback and monitoring," "shaping knowledge," "goals and planning," "repetition and substitution," "antecedents," and "comparison of behaviours."

There were many studies where the authors could not agree on the behaviour change techniques involved within the intervention. The disputed techniques are shown in Table 2. Eight of the 10 studies for which no behaviour change technique code was recorded had potentially included BCTs but the research team could not reach agreement on them. Two studies $[27,56]$ included no discernible BCTs. The most common category where a disagreement occurred between the authors was "goals and planning," with 21 of the 22 studies with a disputed behaviour change technique being discrepant within this cluster. In only 2 of these 21 studies [21, 23] was the discrepancy not within the subcategory "goal setting (behaviour)."

For the studies where it was possible to categorise the type of behaviour change, BCT category according to type of intervention was plotted (Figure 2). While all types of intervention made use of "feedback and monitoring" and "shaping knowledge" techniques physical activity based interventions utilised "comparison of behaviours" and "repetition and substitution" more than dietary or mixed lifestyle

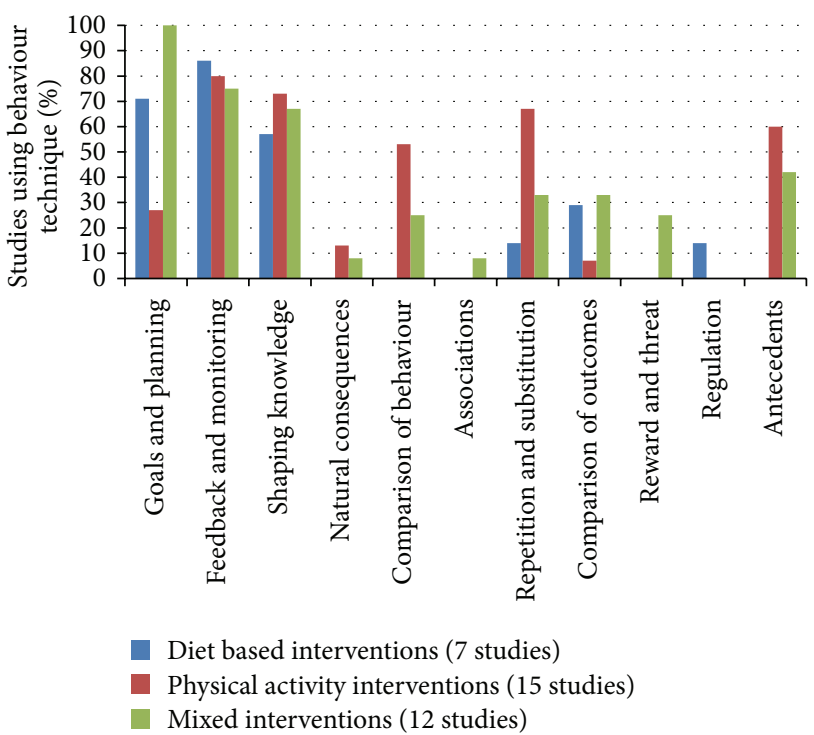

FIGURE 2: Behaviour change technique taxonomy categories according to intervention type ( $n=34$ studies).

interventions. In comparison dietary based and mixed interventions incorporated "goals and planning" more often.

Gestational weight gain was reported in 34 studies; however for 6 of these studies no agreement was obtained for applying a BCT code. The success of each behaviour change technique according to type of intervention in the resulting 28 studies is shown in Figure 3. In studies where a BCT classification could be applied a significant difference in gestational weight gain between the intervention groups and control groups was found more often for diet based $(n=5)$ or mixed interventions $(n=6)$ compared to physical activity based interventions $(n=1)$.

The prevalence of each BCT category in both successful and unsuccessful interventions for reducing gestational weight gain is shown in Table 3. The BCT categories present in $50 \%$ or over of the studies with successful interventions were "feedback and monitoring," "goals and planning," and "shaping knowledge."

\section{Discussion}

We have used the Thangaratinam et al. [3] review as an example of a report incorporating diet, physical activity, and mixed lifestyle interventions with the potential to impact on maternal or fetal weight outcomes. Of the 44 studies included within that review, 34 reported total gestational weight gain.

The most commonly used behaviour change technique categories were "feedback and monitoring," "shaping knowledge," "goals and planning," "repetition and substitution," "antecedents," and "comparison of behaviours." To our knowledge there is only one other study [9] in which lifestyle interventions in pregnancy or the postpartum have been classified according to Michie et al's BCT taxonomy [4]. The behaviour change technique components of interventions in pregnancy aimed at reducing the decline in physical activity were categorised within that study by Currie et al. [9], with 












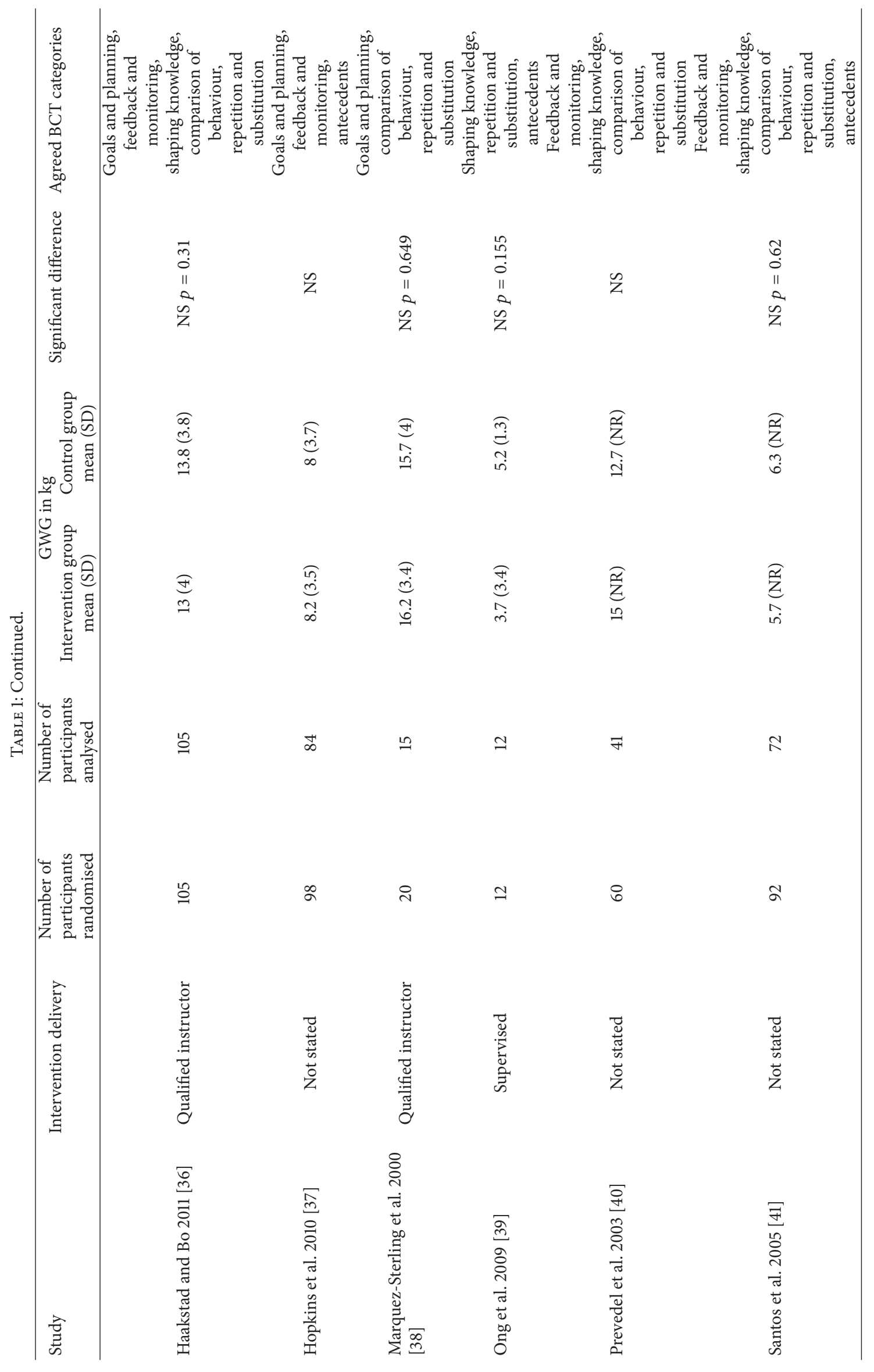

















TABLE 2: Discrepant Behaviour Change Technique categorisation across the studies.

\begin{tabular}{|c|c|c|}
\hline Study & $\begin{array}{l}\text { Discrepant BCT } \\
\text { categorisation }\end{array}$ & $\begin{array}{c}\text { Type of intervention }(\mathrm{D}= \\
\text { diet; } \mathrm{P}=\text { physical activity; } \\
\quad \mathrm{M}=\text { mixed })\end{array}$ \\
\hline Badrawi et al. 1992 [26] & Goals and planning & $\mathrm{D}$ \\
\hline Barakat et al. 2009 [31] & Goals and planning & $\mathrm{P}$ \\
\hline Barakat et al. 2012 [32] & Goals and planning & $\mathrm{P}$ \\
\hline Bechtel-Blackwell 2002 [22] & $\begin{array}{c}\text { Comparison of } \\
\text { outcomes }\end{array}$ & $\mathrm{D}$ \\
\hline Bell and Palma 2000 [42] & Goals and planning & $\mathrm{P}$ \\
\hline Briley et al. 2002 [23] & Goals and planning & $\mathrm{D}$ \\
\hline $\begin{array}{l}\text { Baciuk et al. } 2008 \text { [29] } \\
\text { Cavalcante et al. } 2009 \text { [30] }\end{array}$ & Goals and planning & $\mathrm{P}$ \\
\hline Clapp 1997 [15] & Goals and planning & $\mathrm{D}$ \\
\hline Clapp et al. 2000 [33] & Goals and planning & $\mathrm{P}$ \\
\hline Erkkola 1976 [34] & Goals and planning & $\mathrm{P}$ \\
\hline Erkkola and Makela 1976 [43] & Goals and planning & $\mathrm{P}$ \\
\hline Garshasbi and Faghih 2005 [35] & $\begin{array}{l}\text { Goals and planning } \\
\text { Shaping knowledge }\end{array}$ & $\mathrm{P}$ \\
\hline Lee et al. 1996 [45] & Goals and planning & $\mathrm{P}$ \\
\hline Marquez-Sterling et al. 2000 [38] & $\begin{array}{l}\text { Goals and planning } \\
\text { Shaping knowledge }\end{array}$ & $\mathrm{P}$ \\
\hline Ney et al. 1982 [18] & Goals and planning & $\mathrm{D}$ \\
\hline Ong et al. 2009 [39] & Goals and planning & $\mathrm{P}$ \\
\hline Prevedel et al. 2003 [40] & Goals and planning & $\mathrm{P}$ \\
\hline Rae et al. 2000 [25] & Goals and planning & $\mathrm{D}$ \\
\hline Santos et al. 2005 [41] & Goals and planning & $\mathrm{P}$ \\
\hline Sedaghati et al. 2007 [28] & Goals and planning & $\mathrm{P}$ \\
\hline Wolff et al. 2008 [21] & Goals and planning & $\mathrm{D}$ \\
\hline Yeo et al. 2000 [46] & $\begin{array}{l}\text { Goals and planning } \\
\text { Feedback and } \\
\text { monitoring } \\
\text { Shaping knowledge } \\
\text { Repetition and } \\
\text { substitution }\end{array}$ & $\mathrm{P}$ \\
\hline
\end{tabular}

the 6 most commonly used BCT categories being the same as those found within this study. Others have used Michie's previous taxonomy [8] to code pregnancy and postpartum lifestyle interventions. All of these found behaviour change techniques within the categories of "goals and planning" and "feedback and monitoring" were the most frequently used [5-7]. Hill et al. [6] and Gilinsky et al. [7] both also noted "instruction on how to perform the behavior" was often utilised which sits within the "shaping knowledge" cluster in the Michie et al. BCT taxonomy [4]. Gilinsky et al. [7] also identified "set graded tasks" which is often used in physical activity trials and is classified under the "repetition and substitution" cluster. Hill et al. [6] found studies often provided "information on the consequences of behavior" which corresponds with behaviours in the "natural consequences" cluster. With the exception of Hill et al.s [6] "natural consequences" category, these behaviour change techniques correspond closely with those found in our study.
When assessing BCT taxonomy categories, there were disputes among the authors (Table 2), mostly around the "goal setting (behaviour)" technique. This categorisation was disagreed on for 15 out of the 18 physical activity interventional studies which could account for "goals and planning" appearing to be more often incorporated into dietary based and mixed interventions compared to physical activity interventions. In the majority of these disputed studies there was no explicit reference to goal setting within the descriptions of the intervention procedures provided according to the BCT taxonomy definition: "set or agree on a goal defined in terms of the behaviour to be achieved" [4]. Participants had been assigned to the intervention condition as part of the research protocol. Although the intervention description included exercise classes or similar, it was not clear whether or not a goal had been set or agreed to attend/engage in these classes, even though this seemed likely to have occurred. These disagreements may reflect health psychologists stricter 
TABLE 3: Prevalence of BCT categories within successful and unsuccessful interventions at reducing gestational weight gain.

\begin{tabular}{lcc}
\hline & $\begin{array}{c}\text { BCTs present in successful intervention (\% of 12 } \\
\text { studies) }\end{array}$ & $\begin{array}{r}\text { BCTs present in unsuccessful } \\
\text { intervention (\% of 16 studies) }\end{array}$ \\
\hline Goals and planning & 75.0 & 50.0 \\
Feedback and monitoring & 91.7 & 75.0 \\
Shaping knowledge & 50.0 & 81.3 \\
Natural consequences & 0 & 6.3 \\
Comparison of behaviour & 8.3 & 56.3 \\
Associations & 8.3 & 0 \\
Repetition and substitution & 16.7 & 68.8 \\
Comparison of outcomes & 25.0 & 18.8 \\
Reward and threat & 16.7 & 6.3 \\
Regulation & 8.3 & 0 \\
Antecedents & 25.0 & 50.0 \\
\hline
\end{tabular}

BCT = Behaviour Change Technique.


Unsuccessful

Successful

FIGURE 3: Success of intervention on gestational weight gain across intervention type. 
understanding and interpretation of BCT coding, which does not necessarily match the understanding of clinicians and emphasises the potential difficulties of translating BCT's into practice. Clarification of these ambiguities is required to enhance the implementation and reporting of BCT's in research and practice.

Categories of behaviour change techniques were present in both effective and ineffective interventions, except for "regulation" which was only present in one successful diet based intervention and "association" which was within one successful mixed intervention. Others who have assessed behaviour change techniques utilised within interventions have similarly found behaviour change strategies to be present in both effective and ineffective studies [5]. Within this current study physical activity interventions were largely unsuccessful at managing gestational weight gain, whereas individual behaviour change techniques within diet based or mixed interventions were of varied success. However the success or failure of an intervention could be a result of a number of factors beyond the specific BCT's, for example, the study design, insufficiency of the sample size, or poor fidelity to intervention processes and attrition rates in the original studies.

The success or failure of the interventions may have been influenced by individual BCTs or by the specific combination of BCTs within the intervention. It was not possible to statistically analyse the individual effectiveness of BCTs or to assess the effectiveness of different combinations of behaviour techniques due to the number of different combinations of BCTs present within studies, which is a limitation of this review. However it was noted that successful interventions always included BCTs from one or both of "goals and planning" or "monitoring and feedback". This is in line with Michie et al's [60] findings with regard to healthy eating and physical activity interventions in the general population, with what Gilinsky et al. [7] found for interventions effective at increasing postnatal physical activity and with Harkin et al. [61] who found larger effect sizes in interventions incorporating monitoring of goal progress. When specifically looking at gestational weight gain studies utilising explicit goal setting Brown et al. [14] found a difference in the types of interventions which were effective at different body mass indexes (BMIs) with some interventions working best for women of normal weight and others for women who were overweight or obese. Future research into effective behaviour change techniques will need to take account of potential differential effects across various BMI categories.

The lack of clear and consistent reporting of which behaviour change techniques were undertaken within each intervention was a recurrent theme across this study. Poor reporting, making classification of BCTs difficult, was noted to occur within three main areas: lack of differentiation between the intervention processes and the research processes of the study; difficulties in determining which components were delivered only to the intervention group rather than to both the intervention and control groups; and finally poor or vague definitions of the behaviour change components used. Each of these areas will be discussed in turn.
Some studies were noted to lack clarity over whether the incorporated behaviours were part of the intervention or just part of the study design, for example, glucose monitoring, blood pressure measurements, and completing questionnaires. If these activities were purely for the researchers own benefit to determine clinical outcome measures for the study they would not be part of the intervention and therefore should not be part of the behaviour change technique classification; however if participants were given feedback on the results of blood pressure readings or their current weight in order to promote behaviour change then these procedures would be part of the intervention and their component techniques should be classified This lack of clarity across the studies made BCT classification difficult. The importance of clear reporting was also highlighted due to difficulties in determining which behavioural processes were solely applied to the intervention group. For example, statements such as "participants were weighed at each appointment" did not make it clear if everyone was weighed or just the intervention group.

Behaviour change technique coding was difficult as some studies used vague phrases such as "nutrition counselling" or "education" to describe their interventions and did not clearly specify what techniques these interventions included. Furthermore interventions such as water aerobics sessions or gym access where a fitness instructor was present would most likely include "how to perform the behavior" or "demonstrating the behaviour"; however when this was not explicitly stated it was difficult to identify the techniques and their effectiveness in a standardised and consistent manner. Others have also described the difficultly of applying behaviour change codes to intervention components due to a lack of specificity within reports [5].

One study noted by the authors to provide a clear description which allowed rigorous behaviour change technique codes to be applied was Jeffries et al. [49]. Codes included "goal setting the outcome" as intervention women were informed of their optimal weight gain based on their BMI and Institute of Medicine (IOM) guidelines and given personalised weight gain charts and "self-monitoring the outcome" as intervention group women were asked to weigh themselves every 4 weeks and record it on their chart. In contrast an example of reporting which made BCT classification difficult is Bechtel-Blackwell et al. [22]. They conducted an education based intervention where the intervention group had three 20 minute group sessions which covered: "nutritional needs specific to the woman's stage of her pregnancy." It was not clear whether these sessions just provided information or worked through problems to provide solutions (i.e., if you feel sick, then drink water or go for a walk). No code could therefore be applied.

When developing intervention studies researchers should "clearly define and provide a rationale for all behaviour change techniques that have been included" [62]. Future studies should use frameworks for intervention design such as the Behaviour Change Wheel [63] that guide developers through the process of developing a clear rationale based on evidence. Reporting behaviour change interventions stating what has been done using the standardised terms found 
in the behaviour taxonomy would enable other researchers to understand exactly what the intervention included and would allow statistical analysis to evaluate the effectiveness of specific study components. This would provide a more robust conclusion of the effectiveness of specific BCT categories at preventing excessive gestational weight gain, facilitating the replication of successful interventions or intervention components. The lack of standardised terms in the maternal obesity intervention literature, and the use of vague terms such as "nutrition counselling" means that we cannot understand what aspects of the intervention made it successful and that we cannot properly replicate it in future research. Without the ability to build on knowledge in this way researchers will not be able to improve intervention design in the future.

\section{Conclusions}

Coding interventions using the BCT taxonomy is valuable in the field of gestational weight management. However a better understanding of these techniques, clarity in their implementation, and reporting in a standard format are necessary to allow a robust and reliable evaluation of their efficacy.

\section{Disclaimer}

The views and opinions expressed are those of the authors, and not necessarily those of the NHS, the NIHR, or the Department of Health.

\section{Conflict of Interests}

The authors declare that there is no known conflict of interests.

\section{Acknowledgments}

The authors acknowledge S. Thangaratinam and K. S. Khan for allowing us to use their original review paper. They would like to thank the National Institute for Health Research Collaboration for Leadership in Applied Health Research and Care for Yorkshire and Humber (NIHR CLAHRC YH) for supporting them in conducting this research. Further details about the new NIHR CLAHRC YH can be found at http://clahrc-yh.nihr.ac.uk/.

\section{References}

[1] I. Guelinckx, R. Devlieger, K. Beckers, and G. Vansant, "Maternal obesity: pregnancy complications, gestational weight gain and nutrition," Obesity Reviews, vol. 9, no. 2, pp. 140-150, 2008.

[2] M. Viswanathan, A. M. Siega-Riz, M.-K. Moos et al., "Outcomes of maternal weight gain," Evidence Report/Technology Assessment 168, Agency for Healthcare Research and Quality, Rockville, Md, USA, 2008.

[3] S. Thangaratinam, E. Rogozińska, K. Jolly et al., "Effects of interventions in pregnancy on maternal weight and obstetric outcomes: meta-analysis of randomised evidence," British Medical Journal, vol. 344, no. 7858, Article ID e2088, 2012.

[4] S. Michie, M. Richardson, M. Johnston et al., "The behavior change technique taxonomy (v1) of 93 hierarchically clustered techniques: building an international consensus for the reporting of behavior change interventions," Annals of Behavioral Medicine, vol. 46, no. 1, pp. 81-95, 2013.

[5] B. Gardner, J. Wardle, L. Poston, and H. Croker, "Changing diet and physical activity to reduce gestational weight gain: a metaanalysis," Obesity Reviews, vol. 12, no. 7, pp. e602-e620, 2011.

[6] B. Hill, H. Skouteris, and M. Fuller-Tyszkiewicz, "Interventions designed to limit gestational weight gain: a systematic review of theory and meta-analysis of intervention components," Obesity Reviews, vol. 14, no. 6, pp. 435-450, 2013.

[7] A. S. Gilinsky, H. Dale, C. Robinson, A. R. Hughes, R. McInnes, and D. Lavallee, "Efficacy of physical activity interventions in postnatal populations: systematic review, meta-analysis and content coding of behaviour change techniques," Health Psychology Review, vol. 9, no. 2, pp. 244-263, 2015.

[8] S. Michie, S. Ashford, F. F. Sniehotta, S. U. Dombrowski, A. Bishop, and D. P. French, "A refined taxonomy of behaviour change techniques to help people change their physical activity and healthy eating behaviours: the CALO-RE taxonomy," Psychology \& Health, vol. 26, no. 11, pp. 1479-1498, 2011.

[9] S. Currie, M. Sinclair, M. H. Murphy, E. Madden, L. Dunwoody, and D. Liddle, "Reducing the decline in physical activity during pregnancy: a systematic review of behaviour change interventions," PLoS ONE, vol. 8, no. 6, Article ID e66385, 2013.

[10] I. Streuling, A. Beyerlein, and R. von Kries, "Can gestational weight gain be modified by increasing physical activity and diet counseling? A meta-analysis of interventional trials," American Journal of Clinical Nutrition, vol. 92, no. 4, pp. 678-687, 2010.

[11] H. Skouteris, L. Hartley-Clark, M. McCabe et al., "Preventing excessive gestational weight gain: a systematic review of interventions," Obesity Reviews, vol. 11, no. 11, pp. 757-768, 2010.

[12] E. Oteng-Ntim, R. Varma, H. Croker, L. Poston, and P. Doyle, "Lifestyle interventions for overweight and obese pregnant women to improve pregnancy outcome: systematic review and meta-analysis," BMC Medicine, vol. 10, article 47, 2012.

[13] J. Choi, Y. Fukuoka, and J. H. Lee, "The effects of physical activity and physical activity plus diet interventions on body weight in overweight or obese women who are pregnant or in postpartum: a systematic review and meta-analysis of randomized controlled trials," Preventive Medicine, vol. 56, no. 6, pp. 351-364, 2013.

[14] M. J. Brown, M. Sinclair, D. Liddle, A. J. Hill, E. Madden, and J. Stockdale, "A systematic review investigating healthy lifestyle interventions incorporating goal setting strategies for preventing excess gestational weight gain," PLoS ONE, vol. 7, no. 7, Article ID e39503, 2012.

[15] J. F. Clapp, "Diet, exercise, and feto-placental growth," Archieves of Gynecology and Obstetrics, vol. 260, no. 1, pp. 101-108, 1997.

[16] C. A. Crowther, J. E. Hiller, J. R. Moss, A. J. McPhee, W. S. Jeffries, and J. S. Robinson, "Effect of treatment of gestational diabetes mellitus on pregnancy outcomes," The New England Journal of Medicine, vol. 352, no. 24, pp. 2477-2486, 2005.

[17] M. B. Landon, C. Y. Spong, E. Thom et al., "A multicenter, randomized trial of treatment for mild gestational diabetes," The New England Journal of Medicine, vol. 361, no. 14, pp. 1339-1348, 2009. 
[18] D. Ney, D. R. Hollingsworth, and L. Cousins, "Decreased insulin requirement and improved control of diabetes in pregnant women given a high-carbohydrate, high-fiber, low-fat diet," Diabetes Care, vol. 5, no. 5, pp. 529-533, 1982.

[19] J. A. Quinlivan, L. T. Lam, and J. Fisher, "A randomised trial of a four-step multidisciplinary approach to the antenatal care of obese pregnant women," The Australian and New Zealand Journal of Obstetrics and Gynaecology, vol. 51, no. 2, pp. 141-146, 2011.

[20] Y. S. Thornton, C. Smarkola, S. M. Kopacz, and S. B. Ishoof, "Perinatal outcomes in nutritionally monitored obese pregnant women: a randomized clinical trial," Journal of the National Medical Association, vol. 101, no. 6, pp. 569-577, 2009.

[21] S. Wolff, J. Legarth, K. Vangsgaard, S. Toubro, and A. Astrup, "A randomized trial of the effects of dietary counseling on gestational weight gain and glucose metabolism in obese pregnant women," International Journal of Obesity, vol. 32, no. 3, pp. 495501, 2008.

[22] D. A. Bechtel-Blackwell, "Computer-assisted self-interview and nutrition education in pregnant teens," Clinical Nursing Research, vol. 11, no. 4, pp. 450-462, 2002.

[23] C. Briley, N. L. Flanagan, and N. M. Lewis, "In-home prenatal nutrition intervention increased dietary iron intakes and reduced low birthweight in low-income African-American women," Journal of the American Dietetic Association, vol. 102, no. 7, pp. 984-987, 2002.

[24] J. Khoury, T. Henriksen, B. Christophersen, and S. Tonstad, "Effect of a cholesterol-lowering diet on maternal, cord, and neonatal lipids, and pregnancy outcome: a randomized clinical trial," American Journal of Obstetrics \& Gynecology, vol. 193, no. 4, pp. 1292-1301, 2005.

[25] A. Rae, D. Bond, S. Evans, F. North, B. Roberman, and B. Walters, "A randomised controlled trial of dietary energy restriction in the management of obese women with gestational diabetes," The Australian and New Zealand Journal of Obstetrics and Gynaecology, vol. 40, no. 4, pp. 416-422, 2000.

[26] H. Badrawi, M. K. Hassanein, M. H. H. Badraoui, Y. A. Wafa, H. A. Shawky, and N. Badrawi, "Pregnancy outcome in obese pregnant mothers," Journal of Perinatal Medicine, vol. 20, supplement 1, p. 203, 1992.

[27] T. G. Gomez, J. G. Delgado, A. A. Agudelo, and H. Hurtado, "Diet effects on the perinatal result of obese pregnant patient," Revista Colombiana de Obstetricia y Ginecología, vol. 45, pp. 313-316, 1994 (Spanish).

[28] P. Sedaghati, V. Ziaee, and A. Ardjmand, "The effect of an ergometric training program on pregnants weight gain and low back pain," Gazzetta Medica Italiana Archivio per le Scienze Mediche, vol. 166, no. 6, pp. 209-213, 2007.

[29] E. P. Baciuk, R. I. Pereira, J. G. Cecatti, A. F. Braga, and S. R. Cavalcante, "Water aerobics in pregnancy: cardiovascular response, labor and neonatal outcomes," Reproductive Health, vol. 5, article 10, 2008.

[30] S. R. Cavalcante, J. G. Cecatti, R. I. Pereira, E. P. Baciuk, A. L. Bernardo, and C. Silveira, "Water aerobics II: maternal body composition and perinatal outcomes after a program for low risk pregnant women," Reproductive Health, vol. 6, article 1, 2009.

[31] R. Barakat, A. Lucia, and J. R. Ruiz, "Resistance exercise training during pregnancy and newborn's birth size: a randomised controlled trial," International Journal of Obesity, vol. 33, no. 9, pp. 1048-1057, 2009.
[32] R. Barakat, Y. Cordero, J. Coteron, M. Luaces, and R. Montejo, "Exercise during pregnancy improves maternal glucose screen at 24-28 weeks: a randomised controlled trial," British Journal of Sports Medicine, vol. 46, no. 9, pp. 656-661, 2012.

[33] J. F. Clapp III, H. Kim, B. Burciu, and B. Lopez, "Beginning regular exercise in early pregnancy: effect on fetoplacental growth," American Journal of Obstetrics \& Gynecology, vol. 183, no. 6, pp. 1484-1488, 2000.

[34] R. Erkkola, "The influence of physical training during pregnancy on physical work capacity and circulatory parameters," Scandinavian Journal of Clinical \& Laboratory Investigation, vol. 36, no. 8, pp. 747-754, 1976.

[35] A. Garshasbi and Z. S. Faghih, "The effect of exercise on the intensity of low back pain in pregnant women," International Journal of Gynecology \& Obstetrics, vol. 88, no. 3, pp. 271-275, 2005.

[36] L. A. H. Haakstad and K. Bo, "Exercise in pregnant women and birth weight: a randomized controlled trial," BMC Pregnancy and Childbirth, vol. 11, article 66, 2011.

[37] S. A. Hopkins, J. C. Baldi, W. S. Cutfield, L. McCowan, and P. L. Hofman, "Exercise training in pregnancy reduces offspring size without changes in maternal insulin sensitivity," The Journal of Clinical Endocrinology \& Metabolism, vol. 95, no. 5, pp. 20802088, 2010.

[38] S. Marquez-Sterling, A. C. Perry, T. A. Kaplan, R. A. Halberstein, and J. F. Signorile, "Physical and psychological changes with vigorous exercise in sedentary primigravidae," Medicine and Science in Sports and Exercise, vol. 32, no. 1, pp. 58-62, 2000.

[39] M. J. Ong, K. J. Guelfi, T. Hunter, K. E. Wallman, P. A. Fournier, and J. P. Newnham, "Supervised home-based exercise may attenuate the decline of glucose tolerance in obese pregnant women," Diabetes \& Metabolism, vol. 35, no. 5, pp. 418-421, 2009.

[40] T. T. S. Prevedel, I. M. P. Calderon, M. H. De Conti, E. B. Consonni, and M. V. C. Rudge, "Maternal and perinatal effects of hydrotherapy in pregnancy," Revista Brasileira de Ginecologia e Obstetrícia, vol. 25, no. 1, pp. 53-59, 2003.

[41] I. A. Santos, R. Stein, S. C. Fuchs et al., "Aerobic exercise and submaximal functional capacity in overweight pregnant women: a randomized trial," Obstetrics \& Gynecology, vol. 106, no. 2, pp. 243-249, 2005.

[42] R. J. Bell and S. M. Palma, "Antenatal exercise and birthweight," The Australian and New Zealand Journal of Obstetrics and Gynaecology, vol. 40, no. 1, pp. 70-73, 2000.

[43] R. Erkkola and M. Makela, "Heart volume and physical fitness of parturients," Annals of Clinical Research, vol. 8, no. 1, pp. 1521, 1976.

[44] A. Khaledan, S. Midar, N. S. Motahari Tabari, and M. Ahmad Shirvani, "Effect of an aerobic exercise program on fetal growth in pregnant women," Journal of HAYAT, vol. 16, no. 1, pp. 55-64, 2010.

[45] G. Lee, S. Challenger, M. McNabb, and M. Sheridan, "Exercise in pregnancy," Modern Midwife, vol. 6, no. 8, pp. 28-33, 1996.

[46] S. Yeo, N. M. Steele, M.-C. Chang, S. M. Leclaire, D. L. Ronis, and R. Hayashi, "Effect of exercise on blood pressure in pregnant women with a high risk of gestational hypertensive disorders," Journal of Reproductive Medicine for the Obstetrician and Gynecologist, vol. 45, no. 4, pp. 293-298, 2000.

[47] S. M. Asbee, T. R. Jenkins, J. R. Butler, J. White, M. Elliot, and A. Rutledge, "Preventing excessive weight gain during pregnancy 
through dietary and lifestyle counseling: a randomized controlled trial," Obstetrics \& Gynecology, vol. 113, no. 2, pp. 305312, 2009.

[48] T.-T. Huang, C.-Y. Yeh, and Y.-C. Tsai, "A diet and physical activity intervention for preventing weight retention among Taiwanese childbearing women:a randomised controlled trial," Midwifery, vol. 27, no. 2, pp. 257-264, 2011.

[49] K. Jeffries, A. Shub, S. P. Walker, R. Hiscock, and M. Permezel, "Reducing excessive weight gain in pregnancy: a randomised controlled trial," The Medical Journal of Australia, vol. 191, no. 8, pp. 429-433, 2009.

[50] S. Phelan, M. G. Phipps, B. Abrams, F. Darroch, A. Schaffner, and R. R. Wing, "Randomized trial of a behavioral intervention to prevent excessive gestational weight gain: the Fit for delivery study," American Journal of Clinical Nutrition, vol. 93, no. 4, pp. 772-779, 2011.

[51] B. A. Polley, R. R. Wing, and C. J. Sims, "Randomized controlled trial to prevent excessive weight gain in pregnant women," International Journal of Obesity and Related Metabolic Disorders, vol. 26, no. 11, pp. 1494-1502, 2002.

[52] C. A. Vinter, D. M. Jensen, P. Ovesen, H. Beck-Nielsen, and J. S. Jørgensen, "The LiP (Lifestyle in Pregnancy) study: a randomized controlled trial of lifestyle intervention in 360 obese pregnant women," Diabetes Care, vol.34, no. 12, pp. 2502 2507, 2011.

[53] I. Guelinckx, R. Devlieger, P. Mullie, and G. Vansant, "Effect of lifestyle intervention on dietary habits, physical activity, and gestational weight gain in obese pregnant women: a randomized controlled trial," The American Journal of Clinical Nutrition, vol. 91, no. 2, pp. 373-380, 2010.

[54] A. Hui, L. Back, S. Ludwig et al., "Lifestyle intervention on diet and exercise reduced excessive gestational weight gain in pregnant women under a randomised controlled trial," $B J O G$, vol. 119, no. 1, pp. 70-77, 2011.

[55] A. L. Hui, S. M. Ludwig, P. Gardiner et al., "Community-based exercise and dietary intervention during pregnancy: a pilot study," Canadian Journal of Diabetes, vol. 30, no. 2, pp. 169-175, 2006.

[56] R. A. Jackson, N. E. Stotland, A. B. Caughey, and B. Gerbert, "Improving diet and exercise in pregnancy with Video Doctor counseling: a randomized trial," Patient Education and Counseling, vol. 83, no. 2, pp. 203-209, 2011.

[57] P. Bung, R. Artal, N. Khodiguian, and S. Kjos, "Exercise in gestational diabetes: an optional therapeutic approach?" Diabetes, vol. 40, supplement 2, pp. 182-185, 1991.

[58] A. Ferrara, M. M. Hedderson, C. L. Albright et al., "A pregnancy and postpartum lifestyle intervention in women with gestational diabetes mellitus reduces diabetes risk factors. A feasibility randomized control trial," Diabetes Care, vol. 34, no. 7, pp. 1519-1525, 2011.

[59] P. J. Kulpa, B. M. White, and R. Visscher, "Aerobic exercise in pregnancy," American Journal of Obstetrics and Gynecology, vol. 156, no. 6, pp. 1395-1403, 1987.

[60] S. Michie, C. Abraham, C. Whittington, J. McAteer, and S. Gupta, "Effective techniques in healthy eating and physical activity interventions: a meta-regression," Health Psychology, vol. 28 , no. 6 , pp. 690-701, 2009.

[61] B. Harkin, T. L. Webb, B. P. I. Chang et al., "Does monitoring goal progress promote goal attainment? A meta-analysis of the experimental evidence," Psychological Bulletin, vol. 142, no. 2, pp. 198-229, 2016.
[62] National Institute for Health and Care Excellence, "Behaviour change: individual approaches," NICE Public Health Guidance 49, National Institute for Health and Care Excellence, London, UK, 2014.

[63] S. Michie, L. Atkins, and R. West, The Behaviour Change Wheel: A Guide to Designing Interventions, Silverback Publishing, London, UK, 2014. 




The Scientific World Journal
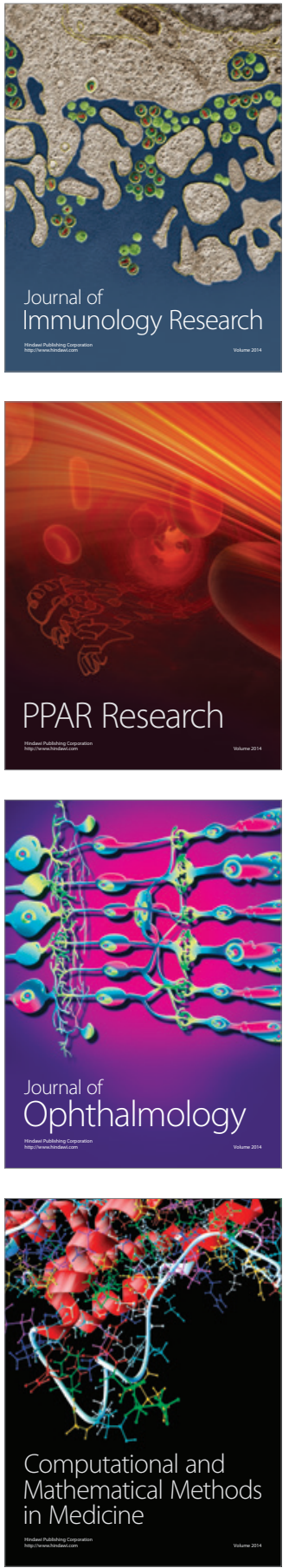



Gastroenterology Research and Practice



\section{Hindawi}

Submit your manuscripts at

http://www.hindawi.com
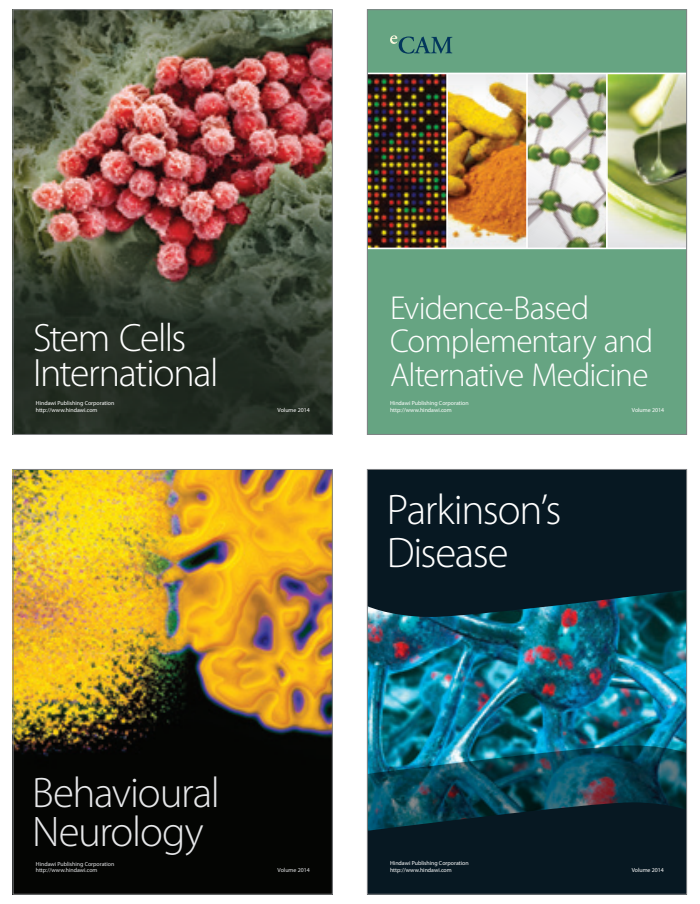


Disease Markers
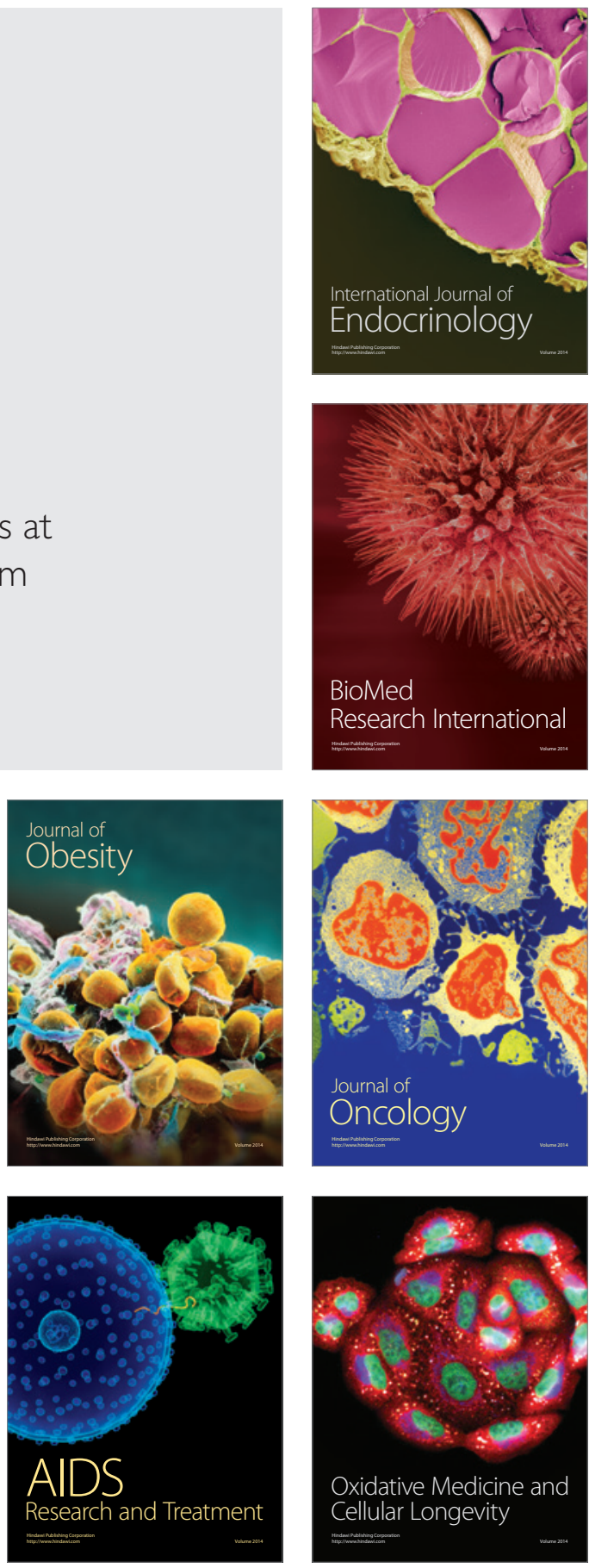